\title{
Supporting Learners' Organization in Collective Challenges
}

\author{
Patrice Moguel $^{1}$, Pierre Tchounikine ${ }^{1}$, and André Tricot ${ }^{2}$ \\ ${ }^{1}$ Lium, Université du Maine, Avenue Laennec, 72085 Le Mans cedex 9, France \\ \{Patrice.Moguel, Pierre.Tchounikine\}@lium.univ-lemans.fr \\ ${ }^{2}$ LTC - IUFM, 56 av. de l'URSS, 31078 Toulouse cedex, France \\ Andre.Tricotatoulouse.iufm.fr
}

\begin{abstract}
This article presents research that aims to construct a computer-based system that (1) supports learners in organizing themselves in the context of a mediated pedagogic collective challenge and (2) supports human tutors in monitoring the learners' process, and supporting it. We present the result of an exploratory experiment that helps in understanding the organizational dimensions of this type of learning situation, a theoretical background originating from $\mathrm{CSCW}$, a prototype designed according to this theoretical background and preliminary results.
\end{abstract}

Keywords: CSCL; Pedagogic Collective Challenge; Organizational Issues.

\section{Introduction}

Collaborative learning emerges from knowledge-generative interactions such as conflict resolution, explanation or mutual regulation [1]. In order to enhance the probability that such interactions occur, CSCL scripts define precise sequences of activities, creating roles and constraining the mode of interaction among peers [2]. Pedagogic collective challenges correspond to another strategy, based on learners' motivation: the scenario is less detailed and, rather, emphasis is on introducing a challenge to enhance learners' motivation for involving themselves and solving the problem.

Scripts and challenges correspond to particular cases of collective work situation, learners being mutually dependent in their work [3]. This requires the overhead activity of articulating their respective activities [4,5]. When learners only communicate via a computer-based system, taking these organizational dimensions into account is a core issue [3,6] as they (1) impact the overall process and (2) conduct learners to be involved in knowledge-generative interactions such as building a common ground, planning, conflict resolution or mutual regulation.

Our research aims at constructing a computer-based system that (1) supports learners in organizing themselves in the context of a mediated pedagogic collective challenge and (2) supports human tutors in monitoring and supporting the learners' process, it. In section 2 we introduce the notion of pedagogic collective challenge. In section 3 we summarize some findings related to an exploratory experiment. In section 4 we present how we use theoretical works by Bardram [4] to model organization. In Section 5 we 
present the prototype we have designed to support learners, and some usability results. Finally, Section 6 presents the results of a preliminary experiment, and Section 7 draws general conclusions and perspectives.

\section{Pedagogic Collective Challenges}

We define a pedagogic collective ${ }^{1}$ challenge as a learning situation where: (1) the problem is designed to make learners practice some target domain-related and/or meta-cognitive competencies; (2) a group of learners is involved, as a team, in the solving of the problem; (3) the solving of the problem requires the learners to join their forces; (4) the problem and the setting are designed to create a positive tension that motivates learners.

Such learning situations present different interesting features [7]: they allow addressing domains (e.g., mathematics) from new perspectives, which can raise the interest of some learners; their playful nature enhances learners' motivation; the fact that they are based on different work modalities (individual, group, class) allow all learners to involve themselves and participate in different ways; the status of «error » is different from usual exercises. Pedagogic challenges present different properties that have been identified as positively impacting students' motivation such as promoting situational interest, students' self determination, working in the presence of others or self regulation $[8,9]$.

Van Eck \& al. [10] highlight that a challenge should be difficult enough to create some uncertainty about obtaining a result whilst not going to an extent where learners may doubt they will succeed. Retroaction is also identified as an important issue for learners' motivation. Attention should be paid to the fact that, when experts tend to spend time in planning their work, novices tend to jump in a too straightforward way to problem-solving basic actions [11].

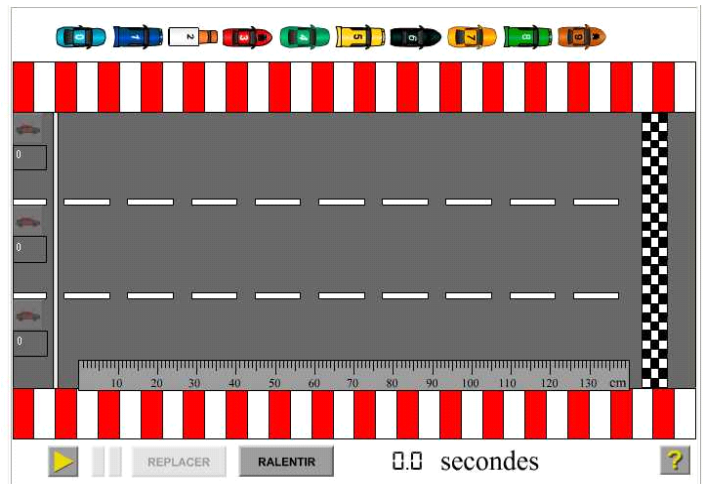

Fig. 1. The simulation (the track) Finally, collective problemsolving is not to be understood as a set of individual (sub)problem-solving and sharing of sub-problems' inputs and outputs [12]. Individual problem-solving strategies can act as resources for proposing what tasks are to be addressed; collective problemsolving however also requires the additional task of the collaboration management.

\footnotetext{
${ }^{1}$ As we believe the "cooperative" / "collaborative" distinction is often a question of level of granularity, point of view and/or matter of concern, we will use "collective" as a wide concept, however using "cooperative" / "collaborative" when well suited or when quoting other authors.
} 
We use the problem (and the Flash simulation) "the race with no winner" created by a community of practice dedicated to the use of simulations in mathematics and physics [13] as a field study. The simulation embeds 10 cars that can be put on a track (cf. Fig 1). The cars have different behaviours: they have different speeds, and when on the track some of them stop for a time (different one from another) and start again. The challenge is as follows: the tutor selects one car and puts it somewhere on the track (not necessarily on the departure line); he then indicates two other cars to the learners; the learners have to calculate where to put each of these two other cars on the track for all three cars to arrive simultaneously at the final line. The pedagogical situation has thus 3 phases: (1) preparing the data: learners must achieve a mathematical analysis of the 10 cars' behaviours and collect the data necessary to establish a relation between the departure position of every single car and its arrival at the final line, which involves calculus and competencies related to position, time and speed (variation rate, table of values, cartesian plan and equation, etc.); (2) the launch of the challenge: the tutor puts a car (out of the 10) on the track, designates two others, and the learners have a limited amount of time to calculate where to put these two cars, on the basis of their behaviours as calculated at the previous step; (3) the simulation is played to check if it is a success or a failure. This can of course be followed by a debriefing phase with the tutor.

Such a simulation / challenge can be used to target different pedagogical objectives, e.g., to discover some principles of physics (relation speed/distance) by inquiry learning, practice the involved calculus techniques or practice how to formalize a problem. In the context of our study, the simulation is used in the context of a mathematical course with 11th Grade students (16-17 year-olds), targeting competencies related to planning and regulation as a means to be able to react to various situations [14,15]: different studies (e.g., [14]) have demonstrated the interest of situations where students are confronted with concrete cases where they have to make different strategies explicit, select one, apply it, and evaluate its effectiveness. Such situations also contribute to active learning by making learners reflect on what they do and by involving them in an evaluation of their process and results. The rationale for using a collective activity is (1) it stimulates making problem-solving strategies explicit and (2) it generates peers' interactions related to the problem-solving strategies and their evaluation (some evaluation thus takes place before and differently from the final simulation). Finally, a minor pedagogical objective is to make learners practice the elaboration and implementation of a mathematical model. With respect to these objectives, the challenge is interesting by the fact that, after the tutor has put his car on the track, the learners only have a limited amount of time to place theirs (in our experiment we gave them 20 minutes). Therefore, in the first phase, they must not only prepare all the useful data (i.e., calculate the different cars' behaviours), but also organize themselves for the second phase: identify what the different tasks to be achieved during phase 2 are (acquire $\mathrm{x}$, measure $\mathrm{y}$, calculate $\mathrm{z}$ ), and decide how to organize themselves (who will achieve each subtask, etc.). As we observed in our exploratory experiment, the learners realize they have enough mathematical knowledge to solve the problem, but also that solving the problem in limited time can be carried out only by sharing the work and adopting an effective strategy. They have to adopt a strategy (out of the different ones they individually proposed) and set up a form of monitoring and regulation of the process. 


\section{Organizational Dimensions of a Pedagogic Collective Challenge}

\subsection{Definition}

A collective learning situation such as a pedagogic collective challenge is made of two overlapping systems of activities: the collective problem-solving and the organization of this collective problem-solving. A pedagogic collective challenge corresponds to a particular case of collective work situation, i.e., a situation where the learners are mutually dependant in their work. Works in CSCW highlight that actors engaged in such interdependent processes must address an overhead activity, that of articulating (dividing, allocating, coordinating, scheduling, meshing, interrelating) their respective activities [4,5]. This is a meta-level overhead activity that is not focused on producing the targeted output, but on setting the conditions of the production of this output by maintaining a more-or-less stable pattern of cooperative arrangement between people.

With respect to organization in the context of CSCL scripts, [3] introduces the notion of learners' self-organization, which is defined as "the meta-level activity that a group of learners engaged in a CSCL script may engage in so as to maintain, within the reference frame that is externally defined by the script, a more-or-less stable pattern of collective arrangement". In this definition, "self" is meant to highlight that, in such a context, part of the organization is set by the script, and part is related to emergent features of learners' enactment of the script at run-time. We use the same notion to analyze pedagogic collective challenges. There is no "obvious" strategy to solve the problem (indeed, there are several good strategies and also bad -ineffectiveones). Learners have some latitude and, in particular, can self-organize themselves (elaborate and consider different strategies). Given the pedagogical objectives, the fact they address this explicitly is of core importance.

\subsection{Exploratory Experiment}

An exploratory experiment was conducted with two groups of three and four learners in a classroom. Each group had a computer with the simulation. The objective of this experiment was to acquire some general input in respect to if and how, in a face-toface setting, learners engage naturally in an organization activity, as a first step to inform the system design. We summarize here below the major findings as they came out from the interactions with the learners, the analysis of the video and of the groups' products.

Learners appear to be very involved in the challenge. They understand what is necessary to cope with. The structure of the challenge (limited duration, mass of data to be collected, crucial precision of measurements, risk of errors and number of calculations to be carried out) does create a situation where learners have to and do interact about the organization to be adopted. In the two groups, the learners naturally came to discuss the strategy and to distribute tasks and roles. We were lucky to observe two different strategies. One of the groups spent quite some time in the planning phase before starting to collect the data. They adopted an organization which could be described as collaborative: the learners remained very close to each other and interacted all along the activity, e.g., two learners measuring a distance to be sure of the value, 
and the third one checking the coherence and structuring the data. The other group adopted a more cooperative division of work, i.e., divided and distributed the tasks and worked in a more autonomous way. No conclusion can be drawn from this, but it can be noted that the first group succeeded when the second failed.

With respect to organizational dimensions, the characteristics of the learners' selforganization that appeared to be positive and important to be transposed for the mediated situation are as follows: spending time to reflect on the strategy; using a common language to describe the data and the actions to achieve; collectively acknowledging the adopted strategy; continuously checking the process with respect to the strategy, and adapting the strategy if needed; easy communication, feeling of proximity and possible mutual assistance. Negative features to be taken into account are as follows. First, the simulation structure may implicitly conduct learners to a simplistic (and rather inefficient) cooperative organization (i.e., dividing the number of cars by the number of students and acting separately). Such a strategy should be allowed, but not implicitly suggested by the setting. Second, not calling the strategy into question during the process is more than risky, in particular given the fact that in case of failure, time is missing to redefine the strategy during the final phase of the challenge. We observed a phenomenon already related in literature: in case of difficulty, if the strategic dimension has always been or has become implicit, the interrelation between learners' actions distends but however does not necessarily conduct them to question their organization. Finally, learners may encounter classical difficulties in collaborating, due to lack of experience. For instance, some learners don't communicate to concentrate better, or for fear of creating a disturbance. At this level, absence of coordination and of information-sharing conducts to persistence of bad strategies, each learner believing he is the only one not knowing what to do or how to do it. From a content-domain point of view (mathematics), two points can be noted: it is important that learners do use a common mathematical language to describe the data in order to understand each other, and they need means to edit the data.

\subsection{Brief Analysis and Conclusions with Respect to Design}

From a general point of view, these findings are in line with general state-of-the-art knowledge in CSCL. Different key-dimensions, in relation to organization, can however be specifically noticed and identified as general specifications for the design of the computational environment.

First, just allowing means to make the strategy explicit is not enough. The system must force learners to create an explicitly shared strategy whose different aspects must be agreed by all members. The system must make the adopted strategy salient during its elaboration and its enactment. It must suggest that each learner has the means and the legitimacy to participate in the elaboration of the strategy, to ask for its reconsideration at any time during enactment, and to participate in its modification. This is in line with findings in CSCW related to the role of plans as being to be thought of as resources (and not constraints) adaptable in context (cf. Bardram's work on the non-contradiction between planning seen from this perspective and Suchman's situated-action views [16]). 
Second, the environment must induce learners to use a common vocabulary to reflect both on organizational issues (e.g., subtasks) and domain-related issues (e.g., data to be acquired). This can be related to the classical notion of common ground [1].

Third, the environment must allow the tutor to detect some aspects of the organization that could lead to breakdowns, and propose means to achieve regulation actions. At the current state of analysis, these aspects are, in particular: the fact that a plan is adopted and collectively acknowledged; the way in which each student participates in the elaboration of the plan; how students share the subtasks; how students achieve their subtasks (e.g., quality of the calculus or the fact that a student does not seem to work any more); deviation in the implementation of the plan (which is not necessarily a problem, but is an interesting point for the tutor); individual or collective demand of a modification of the plan. Identifying these features allow tutor's actions such as: questioning the fairness of the plan; questioning the effectiveness of the plan; supporting the collective planning; supporting a given student for a given task; etc.

Other specifications are: the integration of organizational dimensions, communication dimensions and the simulation; the communication and awareness means.

\section{A Dynamic Model of the Organizational Dimensions of a Pedagogic Collective Challenge}

In line with our conceptualization of pedagogic collective challenges as particular cases of collective work situations [4,5], we adopt an Activity Theory point of view [17]. More precisely, we propose to consider the learners' organizational actions of a pedagogic collective challenge as an activity, and to model it following Bardram's model [4]. This model focuses on collective work dynamics, and stresses the fact that perceiving breakdowns appearing during collaboration is an important dimension of the understanding of the collaboration dynamics.

Bardram proposes a three level structure to denote the transformations that may appear in a collective activity: co-ordination, co-operation and co-construction (cf. Fig 2). He highlights the importance of supporting the dynamic transitions that may occur from one level to another during the activity (these levels corresponding to analytic distinctions: an activity takes place simultaneously at all levels.). At the co-ordination level, actors concentrate on the task they have been assigned. Their work is related to a common goal, but their individual actions are only externally related to each other. They realize the global task from the point of view of their individual activity. Cooperation is an intermediate level where actors are active in considering the shared objective. This enables them to relate to each other and make corrective adjustments to their own and others' actions according to the overall collective objective. Co-construction is the level where actors focus on reconceptualizing their own organization and interaction in relation to

\section{Co-construct ion}

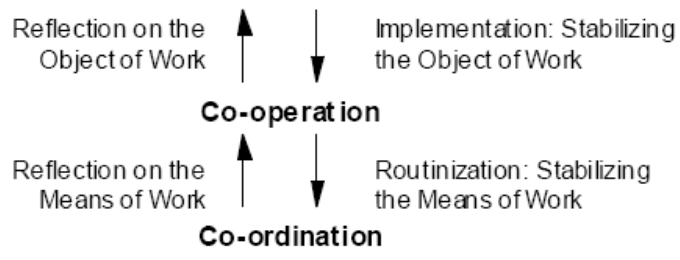

Fig. 2. Bardram's 3-levels model [4] 
their shared objects. Bottom-up transitions are related to an analysis of the object or the means of the work, which can occur in relation to a breakdown or an explicit shift of focus. Top-down transitions are related to the solving of problems and contradictions, and lead to a stabilization of the object and of the means of the work.

In our case, the co-construction level is the level where learners elaborate their strategy. It must correspond to a phase/place allowing the elaboration (and, in case of difficulty or breakdown, revision) of a common view and vocabulary (grounding), and of the general scheduling of subtasks. As said in the introduction, the purpose of such a collective learning situation is to create conditions that enhance the probability that learners will engage in knowledge-generative interactions. Here, drawing learners' attention to this phase, proposing means such as adapted communication tools, and allowing/facilitating tutors' monitoring and regulation, aims at enhancing the chances that students involve in communication, argumentation, analysis or reflection related to both (1) the problem-solving strategy and division of labor and (2) the domain-level issues (mathematical issues). The co-operation level is the level related to how to achieve what has been planned: the role of each member (task attribution, task decomposition if necessary), the means to achieve the tasks (e.g., a tool to help in editing and structuring the data), the sequencing, etc. Organization must be made visible and presented in a way that allows learners and tutors to understand its details. Finally, the co-ordination level is the level where each learner is confronted with his tasks: measuring distance or time, calculating speed, applying mathematical procedures, etc. Tasks, rules or roles have been fixed at the preceding level (and learners can come back to this upper-level by a bottom-up transition). At this level, each learner's work is separated (but coordinated) with that of other learners.

Transitions from one level to another can originate from two sources. First, as suggested by Bardram, learners can spontaneously go from one level to another in relation to a difficulty they encounter, or by a voluntary shift of focus. Such transitions correspond to self-organization dimensions as defined previously. Differently from Bardram's work, in our case another origin for transition appears. We are in a pedagogic context, and the learners' process is monitored by the tutor. He can launch regulation actions, e.g., drawing learners' attention to the fact they should shift from one level to another (i.e., interact about a feature of another level than the current one) in relation to a problem encountered by a learner or by the group, an anticipation of a breakdown, or a pedagogical opportunity.

\section{Prototype and Usability Experiment}

We present here at the same time the prototype and the usability experiment. This experiment was conducted by first testing the individual phase in a classroom, each student working individually on a computer, and the experimenter observing the setting. Then, the collective phases were conducted on-line, two students connecting from home to the server and the experimenter playing the role of the third student online, thus being involved, as an observer, "in" the activity. A second experiment was conducted with three students connected to their individual computer and addressing the overall challenge (all individual and collective phases), which confirmed the results described here after. The prototype is implemented using Flash, PHP, XML and 
network technologies. We focus here on the preparation phases rather than the challenge itself.

The prototype first presents an introduction to the simulation and the challenge. Students then skip to a first individual phase. The system provides each student with the simulation and an array to be filled in individually (cf. Fig 3). This array suggests that students define the data they will need to solve the problem. The first column introduces the basic objects. We have decided to support students by introducing the following notions: "all cars", "cars that stop" and "cars that do not stop". This pedagogic decision implements (on the basis of the lessons learned from the preliminary experiment) the principle "create some uncertainty about obtaining a result whilst not going to an extent where learners may doubt they will succeed" [10]. The second column suggests defining the name of the data to be examined, for instance "car number" or "race duration", and the third column allows a textual description of the data. The last column suggests defining the type of actions related to this data. We support students by providing a predefined list: "read", "measure" or "calculate". Students can add as many lines as they wish.

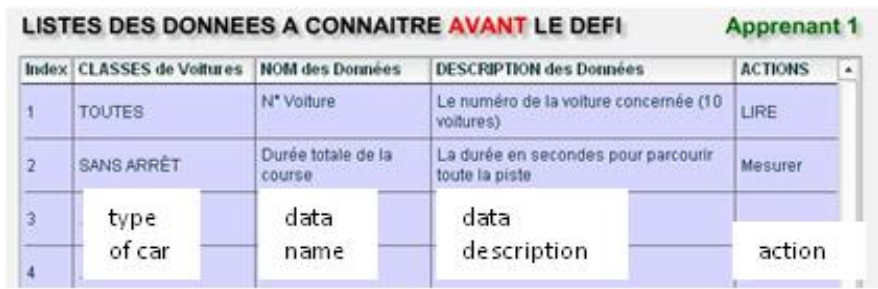

Fig. 3. Defining the data (individual phase)

With respect to the pedagogic analysis and to the theoretical model, this first phase aims at making students familiar with the challenge and the setting. It implements the fact that individual problem-solving strategies can act as resources for proposing which tasks are to be addressed, as an insufficient but preliminary step to the collective solving and, more precisely, the co-construction phase. As expected given the lessons learned from the exploratory experiment, all students first test the simulation and attempt to solve the problem intuitively, by successive trials. At this grade, they however quickly understand that a more analytical approach is requested. Then comes a phase of conceptualization, and in particular the understanding that the requested data and actions should be defined. Following the theoretical background and the lessons learned from the exploratory experiment, the interface supports/suggests this by providing the array to be filled in (at this level different pedagogic options are open: suggesting types of cars and types of actions is but an option). The objective here is to make students appropriate the problem and setting to themselves, and to develop a first individual analysis that will serve as a boot-strap for the building of a common ground and the co-construction phase. Although the focus is on the data to be defined, this data is to be associated with an action (measure, etc.): in fact, each line introduces a subtask. This phase lasted for approximately half an hour. Students then appeared to be willing to discuss the problem collectively, i.e., to skip to phase 2 . 
The next level proposes a shared interface: the simulation, students' individual productions from the first phase (individual arrays), an empty collective similar array, and communication tools: a chat and voting tools to add or suppress a line of the array. The different lines (and their items) must here be collectively defined. Again, types of cars and types of actions are to be selected in a predefined list. The data names and descriptions are to be edited through the shared editor (directly in the array, just by clicking on the cell) and collectively acknowledged by the voting tool: all three students must accept every line, and any of them can come back at any time to what has been defined previously.

With respect to the model, this part of the system corresponds to the coconstruction phase: students define a common view and vocabulary (grounding) and the general plan (sequence of subtasks). From the point of view of the experimenter, it appears that the interface (the structured array) does indeed provide students with what is both a support and a constraint. Due to the similarity with phase 1 interface, students handled this second editor very easily. This is an important point as it was our objective that the collective phases should be as little technically surprising as possible for the students. We were surprised that, although they had a specific chat, they used some of the unused cells of the array to discuss synchronously, each of them using a different cell for typing his/her text. Technically, the chat was much more convenient, in particular because the array only allows 2 lines. This suggests, here again, the relevance of integrated tools. Another organizational pattern appeared: as this phase may become very long, it appeared that students proposed to define a limited time, and to consider that if two students were agreeing on a line, the third should be considered as agreeing if not saying explicitly that he is not after some time. This suggests that overruling functionalities should be available.

The students are then presented with a shared interface for the next two steps (topdown transitions). The interface (cf. Figure 4) is generated from the collective result of the preceding phase. For every couple data/action (e.g., "all cars" / "define race duration") a line is generated with 3 columns for each car (e.g., "car\#0" column "A", "car\#0" column "B", "car\#0" column "C"), "A", "B" and "C" being the names of the students.

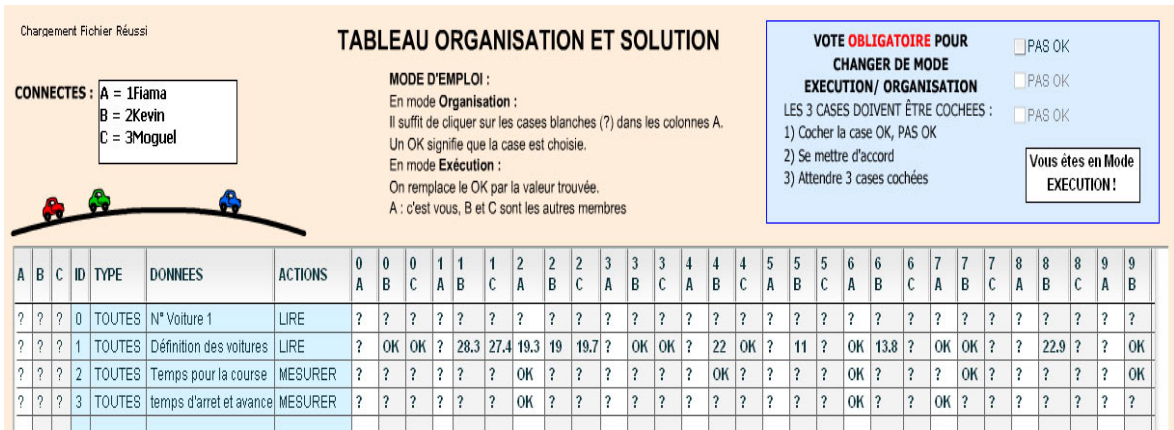

Fig. 4. Defining the organization and enacting the plan 
First, it is suggested that students declare who will achieve each action. All cells are initialized with "?". If " $\mathrm{A}$ " clicks on a cell he declares he will achieve this task; the "?" is replaced by an "OK" in the "A" column; " $\mathrm{B}$ " and "C" are immediately aware as this appears synchronously in the shared array. As each couple car/action is associated with a column by student, the students can decide to delegate each action to just one student, two or three of them. They can come back on their declaration at any time. A chat allows synchronous interaction, and the students have to vote on the result to skip to next phase. During the experiment we could observe propositions such as "important tasks must be done by all (...) and others as measures by only one [student]", counter-propositions, decisions and votes. With respect to the model, we are here at both the co-construction and co-operation levels. The array denotes the (emerging) adopted organization. In the first stage, students were selecting one cell or another more or less randomly (or, more precisely, in a way that was not understandable). After a while, the students' declarations became inter-related. This interrelation denotes another interesting dimension of what we have defined as students' self-organization. This suggests that the interface fulfills the double objective of (1) supporting students and (2) providing the tutor with means to analyze, understand and possibly support the students' organization. How the evolution of the way the array is filled may denote some students' self-organizational dimensions, and how to help the tutor to interpret this, will be two issues at the core of our future investigations.

Second, students arrive at the execution level where they are supposed to enact their plan, i.e., achieve their individual subtasks ("read x", "calculate y", etc.). This idea of "enacting the plan" (to be understood as defined here before) is implemented by providing them with almost the same interface as when defining the tasks (cf. Fig 4). Now, the cells marked as "OK" for a student become editable, i.e., he can edit the value (e.g., "A" measured car\#2 needed 19.3 seconds to complete the race). With respect to the model, we are here at the co-ordination level (top-down transition): students are individually measuring, calculating, etc. As highlighted in Bardram's model, actors concentrate on the task they have been assigned, their work being related to a common goal, but their individual actions being only externally related to each other. The interface is however common, allowing every one to know what he is supposed to do and what the others are doing. The evolution of the solving is denoted by the fact the "OK" are gradually replaced by effective values. Fig 4 denotes the ongoing solving after 30 minutes.

Here again, the coherence organization level / execution level appeared to make the interface easily understandable and usable by the students. Interestingly, and in coherence with the theoretical background, some students did request, during the plan execution (i.e., in context), to skip back to the organization interface and slightly modify the plan. As an example, one of the students discovered some discrepancies in the measures for one of the cars (columns 2A, 2B, 2C) in Fig 4. He used the chat to suggest there was a problem (extracts from the chat, translated: student A "there is a problem ... I think we are not all measuring from the same position ... I'm not measuring from the line but from the start"; student B "I'm measuring from the line ... what do we do?"; observer C "We should all do the same thing"; student B "do we start from the start or from the line"; etc.).

The interface is designed to allow this bottom-up transition: it requires the transition to be an explicit move (because of our pedagogic objectives), but makes it very 
simple. Our concern here is that, as emphasized in the Activity Theory line of thinking, tensions and breakdowns are core phenomena to be studied. Making the bottomup transition explicit makes it perceivable by the students themselves, and by the tutor. In our setting, such tensions and breakdowns may be linked to an ineffective plan, a student that downloads his work, recurrent calculus errors, etc. Tensions and breakdowns in the flow of students' activity might however also originate from inadequate technology and interfaces. This is why we have adopted an iterative design in order to limit this risk, in particular on this very core issue of the connection "defining the subtasks" / "achieving the subtasks". At this level, the important points are: the organization/execution interfaces are similar; if something is modified in the organization the execution interface is automatically adapted; importantly, only the items that have been changed in the organization are modified in the execution interface: modifying the organization does not mean re-starting from scratch or changing everything. Fig 4 also highlights that students can enact partial organizations. As said previously, the different levels are analytic and, in fact, intertwined.

\section{Preliminary Experiment}

In order to prepare an analysis of the impact of the prototype on the way students organize themselves and, in particular, elaborate a definitive analysis grid, we have analyzed (using a first version of such a grid) two groups: group G1 using the prototype and group G2 just using the shared simulation and a basic chat (but no shared array). We focused the analysis on the organizational issues and not the result (as a matter of fact, both groups failed in the final challenge, for different reasons however). We based our analysis on the three distinct aspects of coordination (the degree of mutuality in interaction, the extent to which there was a joint focus of attention and the level of shared task alignment) and the markers proposed in [6]. The results are summarized in Table 1.

Although this is only a very preliminary experiment, we noticed for G1 that the tool inventory data and development of a common language allowed the students to develop a mutual comprehension of the problem, and a general problem solving strategy. The organization tool allowed students to develop a collaborative orientation and a joint problem solving. The challenge itself (final calculations) appeared to be a failure because the group, involved in a very collaborative process, had a (collective) doubt with respect to a given value and spent too much time in discussions to solve it. At the time of the final simulation, they felt as if having correctly managed the preparation phases but having made a punctual mistake, and acknowledging their solution was probably incorrect (which was the case). This confirms that, from a pedagogical point of view, the objective of making students work together and practice the targeted mathematical and organizational competences must not be confused with that of succeeding with the final challenge, and that this must be managed (although teachers may consider the preparation work rather than the result, students are of course very concerned by the final challenge). In G2, each student worked separately, with some communications about the problem comprehension, but little organization. In the final phase the students had three different solutions they had not confronted before the challenge, and no criteria or common understanding to choose one of them. Although 
Table 1. Preliminary experiment analysis

\begin{tabular}{|l|l|l|}
\hline \multicolumn{1}{|c|}{ Criteria } & \multicolumn{1}{|c|}{ Group using the prototype } & \multicolumn{1}{c|}{$\begin{array}{l}\text { Group not using } \\
\text { the prototype }\end{array}$} \\
\hline Duration & 3h 41 & 2h50 \\
\hline Challenge success & Failed but near to success. & Completely failed. \\
\hline Elaborated solution & One solution for the group. & $\begin{array}{l}\text { Three different } \\
\text { solutions. }\end{array}$ \\
\hline Cause of the failure & Too much discussion (cf. "mutuality" item). & Conflicts. \\
\hline $\begin{array}{l}\text { Common view of } \\
\text { the problem }\end{array}$ & Yes & No \\
\hline $\begin{array}{l}\text { Making the } \\
\text { organization } \\
\text { explicit }\end{array}$ & Yes (during 20 minutes and 32 messages). & Very low \\
\hline $\begin{array}{l}\text { Type of } \\
\text { organization }\end{array}$ & Collective, collaborative. & $\begin{array}{l}\text { Individual, } \\
\text { separated. }\end{array}$ \\
\hline $\begin{array}{l}\text { Shared Task } \\
\text { Alignment } \\
\text { (establishment of a } \\
\text { collaborative } \\
\text { orientation toward } \\
\text { problem solving) }\end{array}$ & $\begin{array}{l}\text { High (co-construction of solutions and } \\
\text { feference to others' ideas: collective work } \\
\text { from the beginning to the end). }\end{array}$ & $\begin{array}{l}\text { Low (independent } \\
\text { solution paths and } \\
\text { reference to own } \\
\text { ideas: each member } \\
\text { working separately } \\
\text { on his own } \\
\text { solving). }\end{array}$ \\
\hline $\begin{array}{l}\text { Joint Attention } \\
\text { (degree to which } \\
\text { attention is jointly } \\
\text { focused during } \\
\text { solution-critical } \\
\text { moments) }\end{array}$ & $\begin{array}{l}\text { High (prototype as the centre of } \\
\text { coordination: each member knew exactly the } \\
\text { state of progress of the resolution; joint } \\
\text { monitoring of solution: double check of each } \\
\text { result, etc) }\end{array}$ & $\begin{array}{l}\text { Very low } \\
\text { (individual } \\
\text { monitoring) until } \\
\text { the final phase (the } \\
\text { simulation). }\end{array}$ \\
\hline $\begin{array}{l}\text { Mutuality } \\
\text { reciprocity with } \\
\text { potential for all } \\
\text { members to } \\
\text { meaningfully } \\
\text { contribute) }\end{array}$ & $\begin{array}{l}\text { High (participation of each member in the } \\
\text { elaboration of the solution, data or } \\
\text { explanations; transactional responses and } \\
\text { turn-taking norms respected; resulted in a } \\
\text { common solution, but a punctual mistake } \\
\text { and time/energy to solve it caused the final } \\
\text { failure. }\end{array}$ & $\begin{array}{l}\text { Very low } \\
\text { (conflicting } \\
\text { solutions; no means } \\
\text { to solve the } \\
\text { discrepancies). }\end{array}$ \\
\hline
\end{tabular}

still chatting and willing to solve the problem, they faced a conflict they were not able to solve. At the time of the final simulation they felt as if they were facing a complete failure, and acknowledged their overall strategy was not relevant.

\section{Conclusions and Future Works}

The usability experiments show that the principles we have proposed are relevant for fulfilling the two objectives of (1) supporting students' problem-solving in a way that makes them work out the domain-related and meta-cognitive issues we target and (2) providing tutors with some means to monitor the process. Various ergonomic details of the interfaces were identified and will be improved. However, the overall approach 
and the design decisions underlying the interfaces appear to be adequate in conducting students to work out in an explicit way their organization. The design of the prototype succeeds in making the organizational issues salient (common ground, subtasks, division of labor, top-down and bottom-up transitions) in a way that does not appear too artificial or counter-productive for students while preparing the challenge (on the contrary, it was judged as very helpful). Let us recall that we see this planning and division of work as a resource for both the students and the tutor, and not as a rigid structure (cf. the transition means).

The perspectives include studying the impact of the model/prototype on the students' organization, the relation between the elaborated strategy and winning the challenge, and studying to what extent the elaborated common ground is effectively shared. In particular, we plan to study how the evolution of the way the array is filled in denotes some students' self-organizational dimensions (strategy patterns, tensions and breakdowns, etc.), and how to help the tutor to interpret this and act on the basis of the different types of regulation actions suggested by Bardram's model (suggesting a reflection on the goal of the work or its means in relation to bottom-up transitions, top-down transitions; etc).

\section{References}

[1] Dillenbourg, P.: What do you mean by collaborative learning? In: Dillenbourg, P. (ed.) Collaborative-learning: Cognitive and Computational Approaches, 1999, pp. 1-19. Elsevier, Oxford (1999)

[2] Dillenbourg, P., Tchounikine, P.: Flexibility in macro-scripts for CSCL. Journal of Computer Assisted Learning 23(1), 1-13 (2007)

[3] Tchounikine, P.: Conceptualizing CSCL Macro-Scripts Operationalization and Technological Settings. International Journal of Computer-Supported Collaborative Learning 3(2), 193-233 (2008)

[4] Bardram, J.: Designing for the Dynamics of Cooperative Work Activities. In: Poltrock, S., Grudin, J. (eds.) CSCW conference, Seattle, pp. 89-98 (1998)

[5] Schmidt, K., Bannon, L.: Taking CSCW Seriously: Supporting Articulation Work. CSCW 1(1-2), 7-40 (1992)

[6] Barron, B.: Achieving coordination in collaborative problem-solving groups. The Journal of the Learning Sciences 9(4), 403-436 (2000)

[7] De Vries, E.: Les logiciels d'apprentissage: panoplie ou éventail? Revue Française de Pédagogie 137, 105-116 (2001)

[8] Hidi, S., Harackiewicz, J.M.: Motivating the academically unmotivated: A critical issue for the 21st century. Review of Educational Research 70(2), 151-179 (2000)

[9] Malone, T.W.: Toward a theory of intrinsically motivating instruction. Cognitive Science 4, 333-370 (1981)

[10] Van Eck, R., Dempsey, J.: The effect of competition and contextualized advisement on the transfer of mathematics skills in a computer-based instructional simulation game. Educational Technology Research and Development 50(3) (2002)

[11] Larkin, J.H., Reif, F.: Understanding and teaching problem solving in physics. European Journal of Science Education 1, 191-203 (1979) 
[12] Baker, M.J., Hansen, T., Joiner, R., Traum, D.: The role of grounding in collaborative learning tasks. In: Dillenbourg, P. (ed.) Collaborative Learning: Cognitive and Computational Approaches, pp. 31-63. Pergamon / Elsevier Science, Amsterdam (1999)

[13] http://www.patrickmoisan.net/copains/course_sans_gagnant.html

[14] Poissant, H., Poellhuber, B., Falardeau, M.: Résolution de problèmes, autorégulation et apprentissage. Canadian Journal of Education 19(1), 30-44 (1994)

[15] Flavell, J.H.: Metacognition and cognitive monitoring. American Psychologist 34(10), 906-911 (1979)

[16] Bardram, J.: Plans as situated action: an activity theory approach to workflow systems. In: European Conference on CSCW, Lancaster, UK, pp. 17-32 (1997)

[17] Engeström, Y.: Learning by expanding. An activity-theoretical approach to developmental research. Orienta-Konsultit, Helsinki (1987) 